See discussions, stats, and author profiles for this publication at:

https://www.researchgate.net/publication/300888335

\title{
Theories and methods in the scientific study of consciousness
}

Chapter · January 2015

DOI: $10.1075 /$ aicr.92.02kli

READS

29

4 authors, including:

P. Christiaan Klink

Netherlands Institute for Neur...

29 PUBLICATIONS 219 CITATIONS

SEE PROFILE

Pieter R Roelfsema

Netherlands Institute for Neur...

139 PUBLICATIONS 7,846

CITATIONS

SEE PROFILE 


\section{John Benjamins Publishing Company}

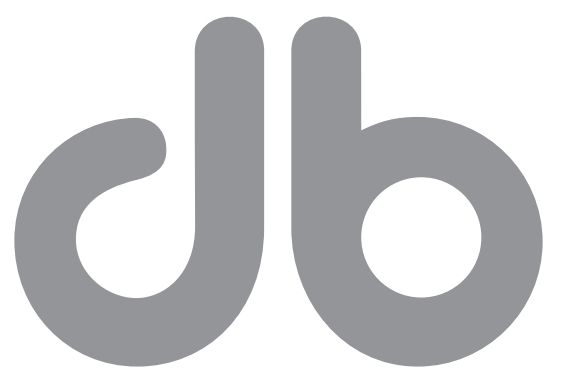

This is a contribution from The Constitution of Phenomenal Consciousness. Toward a science and theory.

Edited by Steven M. Miller.

(c) 2015. John Benjamins Publishing Company

This electronic file may not be altered in any way.

The author(s) of this article is/are permitted to use this PDF file to generate printed copies to be used by way of offprints, for their personal use only.

Permission is granted by the publishers to post this file on a closed server which is accessible to members (students and staff) only of the author's/s' institute, it is not permitted to post this PDF on the open internet.

For any other use of this material prior written permission should be obtained from the publishers or through the Copyright Clearance Center (for USA: www.copyright.com). Please contact rights@benjamins.nl or consult our website: www.benjamins.com Tables of Contents, abstracts and guidelines are available at www.benjamins.com 


\title{
Theories and methods in the scientific study of consciousness
}

\author{
P. Christiaan Klinki,ii, Matthew W. Selfi, Victor A. F. Lammeii \\ and Pieter R. Roelfsemai,ii,iii \\ iNetherlands Institute for Neuroscience / iiUniversity of Amsterdam / \\ iiiVrije Universiteit, Amsterdam
}

\begin{abstract}
The scientific study of consciousness has gained much interest over the past several decades. Here, we provide a primer on the topic by introducing the most commonly used concepts, the most prominent scientific theories, and an overview of broadly used methods of studying consciousness. With a focus on visual perception, we discuss the distinction between phenomenology and cognitive accessibility in the context of the different consciousness theories and highlight elements in which these theories are either complementary or more or less in agreement.
\end{abstract}

\section{Introduction}

Studying consciousness is not a straightforward endeavor. Even when the broad phenomenon of consciousness is narrowed down to visual or perceptual awareness and we assume that it must somehow arise from the brain, there is still a lot of room left to argue. Much of the ongoing debate may however be stemming from the fact that consciousness is a rather poorly defined phenomenon. Most researchers will agree that consciousness is a (neuro)biological phenomenon and that the mechanisms which give rise to consciousness will have to be located in the brain. However, while there are numerous ideas of what constitutes these mechanisms available in the literature there is currently no generally accepted theory of consciousness. In this chapter we will briefly introduce the most influential scientific theories of consciousness, the main points of discussion between them, and the most common experimental paradigms used in the scientific study of consciousness. With a focus on visual perception, this chapter does not aim to provide a complete account of the current state of affairs in the scientific study of consciousness, nor does it aim to resolve any ongoing discussions about the use of specific terminology in consciousness research. Due to space 
limitations, we will also not be able to give a very detailed philosophical account of the conceptual differences between theories of consciousness, nor an advanced overview of the experimental data that supports or opposes the different ideas. Instead, this chapter should rather be regarded as a primer, constructed with the purpose to equip the reader with basic knowledge on the topic and provide a firm starting point for further deliberation and investigation.

\section{Phenomenal and access consciousness}

The scientific problem of consciousness can either be framed in purely biological, in broader cognitive, or in altogether more theoretical philosophical terms. These different approaches are not necessarily mutually exclusive, as they conceptualize aspects of consciousness on fundamentally different levels. A distinction between phenomenal consciousness and access consciousness, for instance, was first introduced in philosophy (Block, 1995, 1996). In this theoretical construct, phenomenal consciousness is equated to raw experiences, or qualia (Chalmers, 1996), i.e., what it is like to experience something (Nagel, 1974). Access consciousness on the other hand relates to the subset of information that is more broadly available to cognitive functions such as giving a verbal report, reasoning, or performing motor actions. This distinction also roughly maps upon the philosophically defined 'hard' and 'easy' problems of consciousness (Chalmers, 1995). Here, the hard problem deals with explaining how phenomenal consciousness or the nature of conscious experience arises from a physical system such as the brain. This is also referred to as 'the explanatory gap' (Levine, 1983). The (relatively) easy problems of consciousness are concerned with explaining cognitive functions commonly ascribed to access consciousness. The contrast between a hard/phenomenal side of consciousness on the one hand, and an easy/ access side of consciousness on the other hand, is either explicitly or implicitly central to most prevailing scientific theories of consciousness. From a biological point of view this has mainly led to a search for either the neural substrates that give rise to consciousness or neural substrates that are specifically involved in some element of conscious processing. Introduced as the search for neural correlates of consciousness (NCC) (Crick \& Koch, 1990, 1998, 2003), this latter approach in particular seems to avoid the hard problem of consciousness altogether. While the term "neural correlate of consciousness" is broadly encountered in the scientific study of consciousness, it is important to realize that there is probably not a single NCC, but multiple ones that depend on the type or the content of consciousness. This becomes clear in the formal definition of NCC, introduced as the minimal set of neuronal events and mechanisms jointly sufficient for a specific conscious percept (Koch, 2004) (Figure 1). Some researchers believe that the accumulation of knowledge about the NCCs related to the easy problem of consciousness may eventually resolve the hard problem (Crick \& Koch, 2003), while others limit the applicability of the search for NCCs to access 

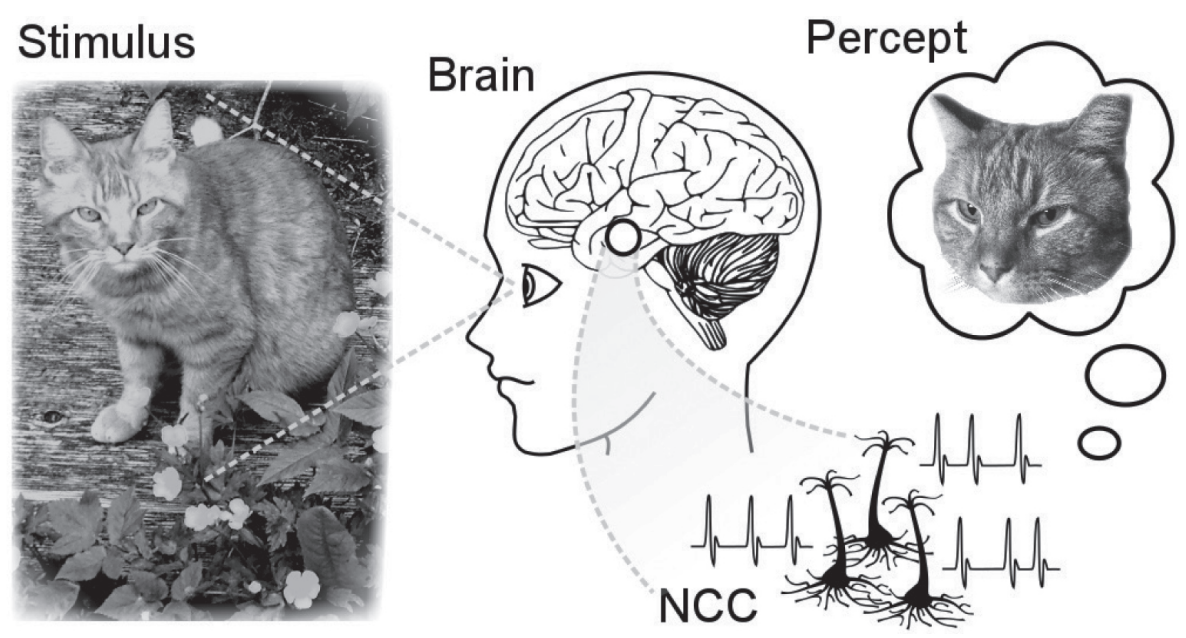

Figure 1. The neural correlates of consciousness (NCC) are the minimal set of neural substrates that are jointly sufficient for a particular conscious percept. In this example, an observer looks at a scene that includes a cat. The neural activity that uniquely corresponds to the conscious percept (here, spiking activity in the inferotemporal cortex) of that cat is the NCC of its perceptual experience. Adapted with permission from Koch (2004).

consciousness (Gaillard et al., 2009), or ignore the distinction altogether for now (Rees, Kreiman, \& Koch, 2002). In addition, as correlations do not necessarily imply causality, NCCs cannot directly tell us anything about the cause, or even more, the constitution of consciousness (Miller, 2007, this volume). This concern has also been articulated as the necessity to look for mechanisms or 'explanatory correlates' of consciousness (Seth, 2009, 2010). It may be possible to partially get around this issue by assigning different NCCs to phenomenal and access consciousness (Block, 2005a; Gaillard et al., 2009; Miller, 2007). Within this subdivision, different phenomenal experiences yield different phenomenal NCCs while access NCCs comprises the neural mechanisms by which experience is made available for further cognitive processing (Block, 2005a). This distinction does however not solve the problem that among the many neural processes that constitute the 'grand neural correlate of consciousness' there may be many that are not directly concerned with creating the content of consciousness but that are either merely facilitative or even a consequence of consciousness. This problem has been recognized and in order to account for it, a distinction has been proposed between neural constituents (i.e., composing elements) of consciousness and neural correlates of consciousness (Miller, 2001, 2007, this volume; Revonsuo, 2001, this volume). This issue of constituents vs. correlates of consciousness is discussed at length on scientific and philosophical grounds throughout this volume. 
Importantly, while a distinction between phenomenal and access consciousness (and their corresponding NCCs) is central to many theories of consciousness, it is not directly clear how this distinction could be investigated. It has indeed been argued that it is impossible to distinguish the raw conscious experience of phenomenal consciousness from the functional availability of access consciousness, simply because the empirical investigation of consciousness by definition requires the product of some sort of cognitive function (Cohen \& Dennett, 2011). This limitation would then restrict the scope of scientific investigation to access consciousness, rendering the concept of phenomenal consciousness useless for any theory of consciousness. Others disagree with this point of view (Lamme, 2010) and stress that a proper theory of consciousness should not only address the subjective experience of a conscious percept, but also and perhaps foremost the constitution of the content of this experience (Fahrenfort \& Lamme, 2012). These opposing viewpoints do however agree that there should at least be two stages of processing involved in the constitution of conscious experience, namely the creation of perceptual content and the conscious experience of this same content. What they disagree on is which of these stages is most interesting in studying the mechanisms of consciousness (Block, 2005b; Cohen \& Dennett, 2011; Fahrenfort \& Lamme, 2012).

\subsection{Consciousness consensus?}

This lack of consensus on what it is precisely that should be studied may be the biggest hurdle in the scientific study of consciousness. The absence of a precise, generally accepted definition of consciousness may confound discussions about the experimental investigation of its neural basis before it has even started. Describing mechanisms of consciousness in neural terms may offer a solution here (Lamme, 2006). On a coarse mechanistic level, for instance, there already seems to be somewhat of a consensus on the sort of neural processing stages that are important for the constitution of conscious visual experience. The flow of visual information through the brain is rather well described by decades of visual neuroscience (Felleman \& Van Essen, 1991; Maunsell \& Newsome, 1987; Price, 2013). In brief, when light patterns hit photoreceptor cells in the mammalian retina they instigate a chain of neural processing that starts within the retina and then moves to the primary visual cortex in the occipital lobe of the brain where it is split into two pathways for further processing. One pathway takes a ventral route into the temporal lobes while the other follows a dorsal route through parietal cortex (Goodale \& Milner, 1992; Merigan \& Maunsell, 1993; Mishkin \& Ungerleider, 1982). Both pathways are connected to frontal lobe regions and motor areas where the visual information arrives in less than 200 ms (Lamme \& Roelfsema, 2000; Tovee, 1994). In addition to this fast forward direction of processing there are also backward projections of information processing. Whenever information reaches a particular functional area it is processed in a specific way and not only fed through to the next 
area along the forward path but also back to previous areas to establish a complex network of recurrent processing (Lamme, Supèr, \& Spekreijse, 1998). Most consciousness researchers will agree with this rough description of visual processing, and most will also subscribe to the idea that whereas all visual information is essentially already present at the level of the retina, additional neural processing is required for conscious experiences to arise. Opinions however start to deviate when you ask where or when along the chain of processing unconscious sensory information becomes a conscious experience. Some theories make an even more subtle subdivision into unconscious/subliminal, pre-conscious and conscious stages (Dehaene, Changeux, Naccache, Sackur, \& Sergent, 2006; Kouider \& Dehaene, 2007) or, as mentioned before, into unconscious, phenomenally conscious and access conscious states (Block, 1995, 1996, 2005a; Lamme, 2003, 2006, 2010). Is this merely a matter of terminology (Block, $\overline{2005 \mathrm{~b}}$ )? Unfortunately not. While the differences between consciousness theories on what is conscious and what is not are derived mostly from philosophical considerations, neural arguments also play a role (Lamme, 2006). Together these differences have strong implications for the practical aspects of the scientific study of consciousness. The brain is an immensely complex organ with processing algorithms that crucially depend on space, time and the type of neural code. Without theoretical focus it will be virtually impossible to study a poorly defined phenomenon such as consciousness in an organ as complex as the brain. Scientific theories of consciousness, guided by dialogues between philosophers and neuroscientists, are therefore crucial in providing the starting points for experimental investigation into the neural nature of consciousness. However, without experimental data to constrain the many theories of consciousness, these dialogues run the danger of turning into mutual monologues where arguments are perpetually repeated and no real progress is made. Fortunately for the field, the required approach of intermingled theoretical and experimental efforts is exactly the road that is currently taken.

\section{From framework to theories}

The renewal of scientific interest in the biological nature of consciousness can to a large extent be attributed to an influential series of publications in the 1990s in which a general framework was proposed to study consciousness experimentally (Crick \& Koch, 1990, 1995, 1998, 2003). While far from being a full-blown theory, this framework introduced a number of concepts and ideas that are now ubiquitous in many theories of consciousness. As an important starting point, the framework suggests to aim our efforts at the visual system, both because of its experimental accessibility and the rich tradition of vision research in neuroscience. The framework further suggests to stay away from the hard problem of consciousness and focus on NCCs, with the hope that an eventual increased understanding of the NCCs will pave the way for a 
sensible approach to investigate the nature of qualia (Crick \& Koch, 2003). Other notable elements of the 'framework for consciousness' are the idea that much of what the brain does happens unconsciously, that the content of conscious experience is determined by competitive interactions between dynamic coalitions of neurons that encode explicit representations of stimulus features, that attention may act as a biasing force in this dynamic competition and gate the content of visual awareness, and that the frontal regions of the brain are somehow essential in establishing conscious experience (Crick \& Koch, 2003; Koch, 2004). Further emphasis is given to the characteristics of connectivity and interneuronal communication, with a distinction between the roles of feedforward and feedback connections as well as different functional roles for different types of neurons or neural coding schemes (e.g., spiking and oscillations). These elements are recurring themes in many of today's most prominent scientific theories of consciousness that we will introduce in the following sections. We will emphasize the potential implementations of these theories in terms of neural processing and their relation with the phenomenal/access consciousness distinction. With this approach we limit our review to scientific theories that have their roots in the known physiology and anatomy of the brain. There are also theories of consciousness that go beyond the brain, for instance by looking for mechanisms of consciousness in large electromagnetic fields surrounding the brain (McFadden, 2002a, 2002b) or by proposing quantum mechanisms in microtubules of the neuronal cytoskeleton as a driving force behind conscious experience (Hameroff, 2006a, 2006b). However, as these theories currently lack convincing evidence or testable predictions (Koch \& Hepp, 2006) they will not be discussed here any further. After introducing the main biologically inspired scientific theories of consciousness, we will go over a number of experimental paradigms that have been instrumental in clarifying neural aspects of consciousness in the past and will likely keep doing so in the future.

\subsection{Global (neuronal) workspace theory}

The global workspace theory (GWT) (Baars, 1993, 1997) is a simple qualitative cognitive theory of consciousness that strongly resembles the mechanism of working memory. It is a contrastive theory that focuses on the distinction between conscious and unconscious processing. Conceptually, unconscious processing is represented by a number of distinct distributed local processes that compete for conscious access. Conscious experience takes shape when widespread activity in these nodes is broadcasted into a global workspace. GWT does not directly map onto the phenomenal/ access consciousness division. In the original interpretation, the information in the ongoing activity of the distributed local nodes is thought to be unconscious, while the content that is broadcasted into the global workspace would correspond directly to access consciousness. This strict interpretation leaves no substrate for phenomenal consciousness and restricts GWT to access consciousness. It is however also possible 
to interpret GWT in a way that allows for an access/phenomenal consciousness distinction. To this end, one has to assume that among the distributed local nodes of unconscious processing there are processes that can potentially be broadcasted into the global workspace and processes that cannot. In this construct, the nodes that cannot broadcast into the workspace remain unconscious, the conglomerate of potential broadcasters constitute phenomenal consciousness, and the few nodes that are broadcasting into the global workspace represent access consciousness (Raffone \& Pantani, 2010). Related to such an interpretation it has been suggested that phenomenal consciousness 'overflows' access consciousness with more information than can be cognitively accessed at any time (Block, 2011). Attention could play a crucial role here as a selection mechanism that picks a specific group of potential broadcasters from the phenomenal-subset of nodes and allows them to give rise to access consciousness.

A neuronal interpretation of GWT (Dehaene, Kerszberg, \& Changeux, 1998; Dehaene \& Naccache, 2001), aptly dubbed global neuronal workspace theory (GNWT), assumes a critical distinction between two classes of functional elements. The first class is formed by a broadly distributed network of more or less specialized local processing nodes of neurons. The activity of these nodes is triggered automatically and their internal computations are performed in isolation from each other, largely outside the scope of consciousness. Such characteristics fit well with the organization of early sensory cortex, where different features are processed in specialized cortical regions (Felleman \& Van Essen, 1991; Grill-Spector \& Malach, 2004; Mesulam, 1998). Visual motion processing, for instance, primarily occurs in a small region of cortex called MT (middle temporal) or V5. Cells in this specific cortical area are however relatively insensitive to color (Born \& Bradley, 2005), whereas neurons in another region known as V4 show the opposite characteristic of being highly sensitive to color, but not so much to motion (Roe et al., 2012). It must however be noted that these functional distinctions are not absolute. In fact, MT-cells are still somewhat modulated by color signals (Seidemann, Poirson, Wandell, \& Newsome, 1999), whereas the activity of V4-cells is also slightly influenced by visual motion (Ferrera, Rudolph, \& Maunsell, 1994). The second class of functional elements in GNWT is formed by the workspace units that are characterized by their extensive connectivity with distant brain areas and mostly located in parietal, cingulate and prefrontal cortex. The broad network of workspace neurons essentially represents access consciousness. In a more recent version of GNWT, the local processing nodes that are not communicating with the workspace nodes are divided into two classes. Nodes that are not activated strongly enough to broadcast their content to the workspace are labeled 'subliminal' and their information will never reach (access) consciousness (Figure 2a). On the other hand, the nodes that are in principle active enough to communicate with the workspace neurons (the potential broadcasters) are called 'preconscious'. They are thought to require an attentional boost to become part of the network that involves the workspace neurons and constitutes (access) consciousness 
(Dehaene, Changeux, Naccache, Sackur, \& Sergent, 2006; Kouider \& Dehaene, 2007). As such, the preconscious nodes can perhaps be thought of as constituting phenomenal consciousness. However, current theories disagree on this interpretation. While the phenomenal/access theory claims phenomenal consciousness as a kind of consciousness (Lamme, 2010), GNWT places the preconscious nodes outside the scope of consciousness by labeling it preconscious (Dehaene et al., 2006).

\subsection{Recurrent processing}

The recurrent processing theory is perhaps the most extensive neurobiological explanation for (visual) consciousness that is explicitly centered on the phenomenal/access separation. First conceived as a potential explanation for the neuronal correlates of figure-ground segregation (Lamme, 1995; Zipser, Lamme, \& Schiller, 1996), the idea of assigning different roles to feedforward, feedback and recurrent processing of visual information has now evolved into a general theory of conscious visual perception (Lamme, 2006, 2010). The basic idea of the theory is that visual information follows a sequential processing path from its entrance into the nervous system at the retina towards output systems such as the motor and prefrontal areas of the brain (Lamme \& Roelfsema, 2000). Within approximately $200 \mathrm{~ms}$, information flows from primary visual cortex to output areas in what is called a fast feedforward sweep (FFS). As the FFS travels up the visual cortical hierarchy, the different cortical regions extract features of the encoded image, filling in an increasing amount of detail and allowing rapid categorization of the visual information. As soon as the feedforward train of information passes a cortical processing station there are additional projections evoked that communicate in opposite and tangential directions through feedback and lateral connections respectively (Figure $2 \mathrm{~b}$ ). Together these non-feedforward connections are called "recurrent" and in the absence of a rapidly following second FFS that acts as a mask they are an automatic consequence of the FFS. Crucial elements in the recurrent processing theory of visual consciousness are the type of processing, feedforward versus recurrent, and the depth of processing.

Not all stimuli will evoke a FFS that goes all the way up to output areas to influence behavior. Due to competition, many features or stimuli that are represented in the earlier processing areas will not be represented in later stages. The role of attention and its relation to consciousness is debated, but in one view of recurrent processing theory, attention is thought to play a crucial role in the competition between these features by gating stimulus information, either by boosting the strength of their representation (Carrasco, Ling, \& Read, 2004; Treue, 2003), or by active gating of the feedforward processing stream (Bosman et al., 2012; Hopfinger, Buonocore, \& Mangun, 2000). With such an interpretation, attention is distinct from consciousness and analogous to the depth of processing. Combining the proposed role of attention with the feedforward-recurrent dichotomy, four distinct stages of processing 
have been identified (Lamme, 2003, 2006). First, superficial feedforward processing involves activity that remains in earlier cortical stages due to a lack of attention and active masking. Second, deep feedforward processing is used as a term to denote feedforward processing that goes all the way up the cortical hierarchy to prefrontal and motor areas because the corresponding stimulus is attended. Crucially however it does not evoke recurrent processing, for instance because of a mask, and therefore remains invisible/unconscious. Third, superficial recurrent processing can be evoked by an unattended stimulus that is not masked. Recurrent processing for these stimuli remains limited to early processing stages. Finally, deep or wide recurrent processing is caused by unmasked, attended stimuli that are processed all the way up to output and evoke a wide pattern of recurrent activity along the way. The central idea is that recurrent processing is a prerequisite for any type of consciousness, but that the depth of recurrent processing distinguishes phenomenal from access consciousness with superficial recurrent processing in early areas corresponding to phenomenal consciousness and deep recurrent processing including frontoparietal brain regions denoting access consciousness (Lamme, 2006, 2010). This is precisely where this interpretation of recurrent processing theory deviates from global neuronal workspace theory: it asserts that even without the inclusion of frontoparietal access networks, there can exist consciousness in the form of a phenomenal visual experience but observers are simply not able to report it. In GNWT this state corresponds to the activation of preconscious nodes, yet these are labeled unconscious. Again, it all depends on the interpretation of the term 'conscious'. GNWT takes an introspective approach and claims that visual information is only conscious when it is accessed and reported, whereas the recurrent processing theory takes a neurobiological point of view and states that if the information is present in the brain and it could in principle be accessed for report, it is already conscious. A detailed philosophical account of the nature of phenomenal consciousness can be found elsewhere in this volume (see Drayson, this volume).

The distinction between attention and consciousness is not undisputed (see also Section 3.6 in this chapter), and other theories of recurrent processing propose that recurrent processes correspond to what psychologists call "top-down" or "task-driven" attention (Lamme \& Roelfsema, 2000; Roelfsema, 2006). In this view, consciousness and attention refer to the same phenomenon because they both depend on recurrent processing. In addition to the debate about the precise relation between attention and consciousness, the strict identification of recurrent processing with consciousness is also not universally supported. Crucially, because any stimulus that is represented in sensory cortex, even those that remain unconscious, will undergo some 'local' recurrent processing by the reciprocal connections between the cortical layers, it remains to be specified how much recurrent processing is required for a stimulus to reach awareness. 

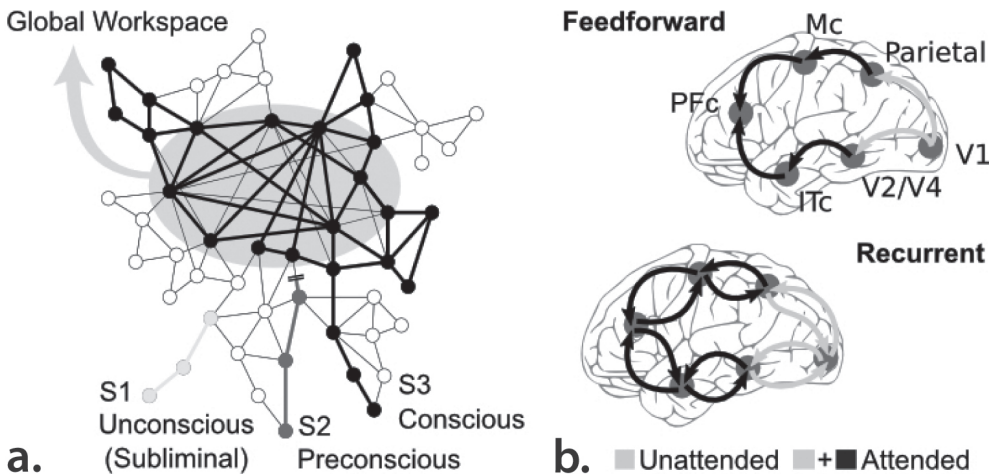

b. Unattended $\square+\square$ Attended

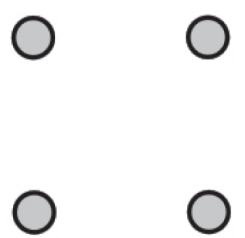

C.

$\Phi=0$

Ventral stream 'What' pathway Vision for perception

d.

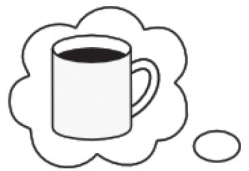

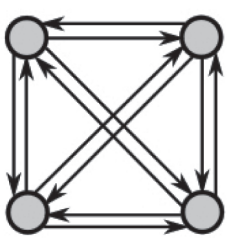

Low $\Phi$

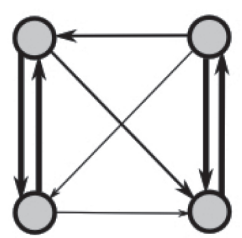

Higher $\Phi$

Figure 2. Theories of consciousness. (a) In global workspace theory, stimuli are consciously perceived if the sensory neurons that encode their features become part of a broad network of interconnected neural assemblies (the global workspace). For this to occur the neural representation of a stimulus should be both strong enough and attended (S3). Stimulus representations that are too weak (S1) cannot connect to the workspace regardless of the attentional state. They remain unconscious/subliminal. Preconscious representations are strong enough yet unattended (S2). They can in principle connect to the workspace if attention is shifted towards them. Adapted with permission from Dehaene et al. (2006). (b) In the latest rendition of the recurrent processing theory, the 'quality of consciousness' depends on the type of neural processing and the attentional state. Stimuli that are only processed in a feedforward manner (e.g., because they are masked) remain invisible. If they are attended they may however still evoke neural processing in 'higher' cortical areas. Consciousness crucially depends on the presence of recurrent processing. Unattended stimuli will only evoke local recurrent processing and give rise to phenomenal consciousness whereas attended stimuli ignite a broad network of recurrent processing leading to access consciousness. (PFc, prefrontal cortex; 


\subsection{Essential nodes of microconsciousness}

Both the recurrent processing theory and the GNWT make a distinction between unconscious processing in local neuronal nodes and engagement of a broader neural network as a requirement for consciousness. The theory of micro-consciousnesses (Zeki, 2001, 2008; Zeki \& Bartels, 1998a, 1998b) takes a radically different approach. It proposes that there is no such thing as a single unified visual consciousness, but that the total of conscious experience is instead made up from a larger number of featureselective so-called essential nodes that are distributed in time and space and that are by themselves sufficient to cause awareness of the feature they are selective for if their activity levels are high enough to pass a certain consciousness-threshold. The information from the essential nodes is then thought to 'postconsciously' bind together into a coherent single perceptual experience called macroconsciousness. Finally, the microconsciousness theory postulates a third level of consciousness dubbed unified consciousness where the content of macroconsciousness becomes available to linguistic and communication systems. The basic idea of spatially separated essential nodes representing not only the processing but also the perceptual site of a visual feature is, similarly to the processing nodes in GWT, inspired by the known architecture of the visual cortex. As we noted before, different visual features are indeed selectively encoded in different regions of the visual cortex, e.g., color in V4 and motion in MT/ V5. Experiments have furthermore shown that humans are generally a few tens of milliseconds earlier aware of color than they are of visual motion (Arnold, Clifford, \& Wenderoth, 2001; Moutoussis \& Zeki, 1997a, 1997b), favoring a temporal distinction between microconsciousnesses. Within the microconsciousness theory, phenomenal consciousness can be assigned both to each essential node separately (feature awareness) and to macroconsciousness as a whole (perception of objects and scenes). Access consciousness on the other hand would then be restricted to the executive output systems at the third level of unified consciousness.

Mc, motor cortex; ITc, inferotemporal cortex). (c) The information integration theory equates consciousness with information integration in complex systems and quantifies it with phi $(\Phi)$. A neural system of unconnected nodes (top) has a $\Phi$ of zero because it cannot integrate the information of the individual units. A system in which every unit is equally strongly connected to every other unit (middle) will have a low $\Phi$ because there is little or no specialization possible. Highest $\Phi$-values are obtained with systems that are highly connected in a heterogeneous way (bottom). Such systems allow the existence of specialized, strongly connected subsystems (the left and right pairs in this example) while maintaining the potential for broader information integration. (d) The duplex vision theory assumes different roles for the dorsal and ventral processing pathways. While the two pathways do exchange information, activity in the ventral ('what') stream is thought to promote conscious perception (e.g., seeing a coffee mug) while activity in the dorsal ('how') stream is used for motor planning (e.g., grasping the mug). 


\subsection{The reentrant dynamic core and information integration theory}

The theory of microconsciousness is perhaps the most localist account among the neurobiological theories of consciousness. On the other end of the spectrum, the reentrant dynamic core theory (RDCT) takes an extreme globalist stand by assigning consciousness to a wide range of dynamic interactions between groups of neurons within the network that connects nuclei in the thalamus with regions of cortex (Edelman, 1993; Edelman \& Tononi, 2000; Tononi \& Edelman, 1998). The hypothesis follows from a more general framework of brain functioning called 'neural darwinism' or 'neuronal group selection theory' (Edelman, 1987; Edelman \& Mountcastle, 1978). In this framework, brain functioning depends on three stages of selection. The first stage is the genetic and epigenetic establishment of basic heterogeneous connectivity patterns during early development. As life progresses, these primary connections are in continuous competition, allowing synaptic reinforcement through experience and behavior. In the final stage called 'reentry', massive parallel networks of reciprocally connected thalamocortical neuronal ensembles (the dynamic core) allow spatiotemporal coordination of neural activity and give rise to coordinated brain function. RDCT does not say much about unconscious brain processes, but it does propose that the integration of information from different brain regions through reentrant coupling of neuronal ensembles is necessary for consciousness to arise. The idea of a dynamic core is reminiscent of the earlier discussed global workspace. It has indeed been argued that the two theories may complement each other in such a way that the dynamic core essentially provides the neuronal substrate for the global workspace (Edelman, Gally, \& Baars, 2011). Like GWT, the RDCT does not directly map upon the phenomenal/access distinction. However, the momentary perceptual experiences arising from integrated reentrant activity in the dynamic core that are labeled primary (sensorimotor) consciousness or 'the remembered present' are akin to the idea of phenomenal consciousness. Primary consciousness is furthermore contrasted with a higher-order consciousness that requires language, allows verbal reports, and thus somewhat resembles access consciousness.

The concept of information integration that is central to the dynamic core hypothesis is further formalized in the information integration theory (IIT) of consciousness (Tononi, 2004). This theory is built around the notion that consciousness is both differentiated and integrated. Differentiation means that any specific momentary conscious state is different from the many other potential states. The integrated aspect on the other hand, refers to the large amount of information about different features that comprises such a conscious state (e.g., the shape and color of an object) and in which the information of one feature cannot be experienced separately from that of another. The analogy of a digital camera may clarify this idea (Tononi, 2008). The sensor chip of such a camera consists of numerous small photodetectors that each carries a binary signal. With one million of such detectors, the camera as a whole has an information encoding capacity of $21,000,000$ bits, which allows the construction of a huge number of unique images. The camera is however not considered conscious 
because the individual detectors are completely independent of one another preventing the integration of all this information. Humans on the other hand do integrate the different features that make up a visual scene, allowing us to become aware of it. IIT uses this principle to quantify consciousness based on a system's information integration capacity (Tononi, 2008; Tononi \& Koch, 2008). This measure, labeled $\Phi$ (phi) and measured in bits, uses information theory to calculate the capacity for information integration based on the complexity and entropy of the system. With IIT it is thus theoretically possible to calculate the amount of consciousness of a complex system. In practice, this turns out to be a task of inconceivable proportion for a large biological system like the brain. To illustrate the basic idea, imagine a hypothetical organism with a brain consisting of only a few neurons. If these neurons are not connected to each other, there will be no integration of the information carried by individual cells and $\Phi$ will be zero. Connecting each neuron to all other neurons does allow information integration, but as everything that happens in such a network is interconnected there is no differentiation and $\Phi$ is not raised very much. The highest possible $\Phi$ for such an extremely simple system will be obtained with an intermediate solution where units are clustered in specialized assemblies of strongly interconnected cells while the assemblies themselves are more sparsely connected (Figure 2c). In the end, $\Phi$ depends on the number of individual units, assemblies and their connectivity patterns (Tononi \& Sporns, 2003). For the human brain, sporting billions of neurons, trillions of connections and an unidentified number of functional assemblies, $\Phi$ cannot be calculated with today's technology but the basic computational principles already lead to some interesting ideas when they are applied to smaller, simplified systems (Oizumi, Albantakis, \& Tononi, 2014). Recurrent processing between functionally specialized nodes for instance would significantly increase $\Phi$ compared to a system where the same nodes are operating in isolation from each other. Furthermore, within IIT there could be many parallel functional networks with a relatively low $\Phi$ that constitute broad phenomenal consciousness while a smaller number of networks with higher $\Phi$ represent access consciousness. It could perhaps even be the case that the reason why these access networks have higher $\Phi$ is because they are (recurrently) connected to an additional output system. This is of course all very speculative, but it illustrates the potential of the IIT perspective to capture the phenomenology of consciousness in a firm computational framework (Oizumi et al., 2014) and unify other, more biologically inspired, theories of consciousness.

\subsection{Duplex vision theory: Action and perception}

The existence of two major brain pathways along which visual information is processed has given rise to the duplex vision theory (DVT) (Goodale, 2004; Goodale \& Milner, 1992; Goodale, Milner, Jakobson, \& Carey, 1991; Milner \& Goodale, 1993). Beyond the primary visual cortex feedforward processing of visual information occurs either in an inferotemporal direction (ventral pathway) or a posterior parietal direction (dorsal 
pathway). The best known functional separation that maps onto these processing pathways is that of specific 'what' and 'where' pathways (Mishkin \& Ungerleider, 1982). This division is based on the features that are processed in areas along the two pathways. Areas that make up the ventral pathway are predominantly concerned with the processing of object features such as shape and color, whereas areas along the dorsal pathway are primarily involved in the representation of spatial aspects such as motion direction and speed. The DVT takes a different approach and classifies the pathways not on input characteristics, but on the type of output system with which they are linked. Information that is processed along the ventral stream is thought to be used by perceptual output systems and give rise to conscious experience whereas information in the dorsal stream is used for automatic visuomotor acts (Goodale \& Milner, 1992). This distinction led to the nomenclature in which the ventral pathway is labeled the 'what', or 'vision for perception' stream and the dorsal pathway the 'how' or 'vision for action' stream (Figure 2d). Under normal circumstances the what and how pathways work together in establishing adaptive behavior but patient studies reveal that they can also work in isolation, i.e., objects may be perceived without observers being able to coordinate motor actions in response to them (Pöppel, Held, \& Frost, 1973; Weiskrantz, Warrington, Sanders, \& Marshall, 1974), and similarly observers may not be able to recognize an object while their corresponding motor actions remain adequate (Goodale et al., 1991; James, Culham, Humphrey, Milner, \& Goodale, 2003).

\subsection{Attention and consciousness}

The relation between attention and consciousness is an ongoing topic of discussion (Dehaene et al., 2006; Koch \& Tsuchiya, 2007, 2012; Lamme, 2004; Marchetti, 2012; Posner, 1994; van Boxtel, Tsuchiya, \& Koch, 2010a). Attention plays a role in many theories of consciousness, sometimes as a biasing or gating factor that influences the content of conscious experience and sometimes as a prerequisite for consciousness to occur altogether. While there seems to be consensus about the fact that consciousness and attention are closely related, some scholars go a step further and propose that attention is a fundamental requirement for consciousness or even the exact same thing (Cohen, Cavanagh, Chun, \& Nakayama, 2012; Jackendoff, 1996; Merikle, Smilek, \& Eastwood, 2001; Posner, 1994, 2012). The potential conflation of consciousness and attention is not helped by the fact that even though both concepts may be intuitively accessible, they both lack a universally accepted definition. From a theoretical point of view, one may argue that attention is a mechanism and consciousness a state, which would make the two concepts of a fundamentally different nature ruling out the possibility that they are the same. This does however not mean that they can easily be distinguished experimentally. Multiple studies in the past few years have specifically been designed to dissociate attention and consciousness and resolve this issue (Cohen et al., 2012; van Boxtel, Tsuchiya, \& Koch, 2010b; Watanabe et al., 2011), but they have not managed to silence the discussion completely. In order to truly disentangle the 
biological bases of attention and consciousness, if this is even possible, we may need to return to more neurobiological approaches of the issue instead of relying on the many existing behavioral paradigms alone. An in depth discussion of the relation between attention and consciousness can be found elsewhere in this volume (see van Boxtel \& Tsuchiya, this volume).

\section{Paradigms used to study consciousness}

There are numerous paradigms available to study consciousness. Here we will focus on studies of visual awareness that have addressed the key question: "What are the differences between neural activities that lead to awareness of a visual stimulus and those that do not?" It is clear from this question that the awareness of the stimulus needs to be varied whilst simultaneously measuring some aspect of its neural representation. The most obvious approach to achieve this aim would be to vary the intensity (e.g., luminance, contrast, duration etc.) of a stimulus and to have observers report their awareness. The measure of neural activity, be it single-unit activity, blood level oxygen dependent signal, or event-related potential could then be sorted according to whether the observer was aware of the stimulus. This simple approach suffers from two key problems: firstly it is unclear how awareness should be measured and secondly it is difficult to dissociate changes in neural activity that were due to changes in the physical properties of the stimulus from changes that were due to the difference between the aware and non-aware states.

Historically, awareness of a stimulus has been measured using approaches ranging from subjective verbal reports to forced-choice approaches where performance is objectively assessed. Subjective approaches suffer from the problem that observers are free to set their own criteria as to when they report being aware of a stimulus. Different observers may choose very different criteria making assessment of the aware/ non-aware state difficult. Forced-choice paradigms on the other hand suffer from the problem that the observer is forced to categorize their phenomenal experience into one of two (or more) categories, which may not reflect their underlying experience. Forced-choice paradigms also suffer from the less obvious problem that participants can perform better than chance for stimuli of which they claim not to be aware. Most famously, patients who display 'blindsight' are able to perform well on certain visual tasks while claiming to be unaware of the stimulus (Pöppel et al., 1973; Pöppel, Brinkmann, Von Cramon, \& Singer, 1978; Stoerig \& Cowey, 2007; although see Zeki \& ffytche, 1998).

This result raises an interesting issue; how can we know whether a blindsight patient is truly unaware of the visual stimulus? For example, patient GY, who is clinically blind in his right hemifield, is able to correctly discriminate the direction of motion of fast-moving stimuli presented in his blind hemi-field, while claiming that he had no awareness of these stimuli (Weiskrantz, Barbur, \& Sahraie, 1995). If we use the 
objective criteria of his performance on the motion discrimination task then we would classify GY as being aware. On the other-hand, if we trust the verbal report then we would classify him as unaware. To provide stricter measures of awareness, studies have moved from using forced-choice discrimination questions (e.g., was it moving left-orright?) to detection tasks (e.g., was the stimulus present or not?). The disadvantage of discrimination tasks is that even very weak forms of neural activity, which may not normally lead to awareness of the stimulus, may be sufficient to discriminate between two forced-choice alternatives. Using detection tasks with high numbers of catch-trials in which the stimulus is absent, and presenting the stimuli in random orders with random timings can provide a measure of awareness, which matches more closely the ability of the patient to subjectively report awareness of a visual stimulus. However, the disadvantage of detection tasks is that the patient is free to set their own criterion level at which to respond that they have seen the stimulus. A patient that is biased or reluctant to report a weak sensation as being aware may show poor performance on detection tasks and good performance on discrimination tasks. A possible solution to this conundrum is to use signal detection theory. Using signal-detection theory it is possible to derive a measure of sensitivity $\left(d^{\prime}\right)$ that is independent of the patient's response bias and decision criterion (Macmillan \& Creelman, 1991). Such an analysis was carried out by Azzopardi and Cowey (1997) who determined that GY shows higher sensitivity on discrimination tasks compared to detection tasks.

Other studies have taken a different approach in which the report of a participant is accompanied by a confidence rating or wager on the correctness of the response (Galvin, Podd, Drga, \& Whitmore, 2003; Kolb \& Braun, 1995; Kornbrot, 2006; Kunimoto, Miller, \& Pashler, 2001; Maniscalco \& Lau, 2012; Persaud, McLeod, \& Cowey, 2007). These studies have utilized the logic that if subjects are aware of a stimulus then there should be a good correlation between their objective performance and their (trial-by-trial) confidence in whether their decision was correct. Conversely if blindsight patients are truly unaware of the visual stimulus then they should show little correlation between their performance on a detection/discrimination task and their confidence about their decision. Applications of signal detection theory to confidence judgments have allowed researchers to generate separate sensitivity measures for the primary task (Type-I sensitivity) and the ability of subjects to judge the correctness of their responses (Type-II sensitivity) (Galvin et al., 2003; Maniscalco \& Lau, 2012). This measure of sensitivity has been suggested to capture the 'meta-cognitive' abilities of subjects, that is, the ability to make a judgment about one's own awareness. High meta-cognitive ability has been proposed as a hallmark of a conscious process and dissociations between perceptual abilities and meta-cognitive abilities could signal evidence for perception without awareness (Ko \& Lau, 2012; Persaud et al., 2007).

Despite the advances discussed here it remains difficult to assess whether someone was aware of a visual stimulus based purely on behavioral reports. The approaches we discuss below typically avoid this problem by using stimulus configurations for which observers are both subjectively and objectively unaware of aspects of the stimulus. 


\subsection{Paradigms to study the NCC}

A key approach to studying visual awareness that was outlined by Crick and Koch (2003) is to dissociate patterns of neural activity that are the result of changes in the visual stimulus from those that are due to changes in awareness. To achieve this goal it is important that the physical stimulation of the retina is held constant across different awareness conditions (Kim \& Blake, 2005). The companion to this volume (Miller, 2013) extensively describes what is perhaps the most well-known example of such a paradigm - binocular rivalry (Blake \& Logothetis, 2002). In binocular rivalry the retinal input is held constant and the observer's awareness spontaneously fluctuates between different states. Changes in neural activity observed between these different states can therefore not be attributed to the stimulus itself but should be assigned to some aspect of the change in awareness. Such paradigms have perhaps provided the most revealing insights so far concerning the NCC.

\subsection{1 (Continuous) Flash suppression and motion-induced blindness}

While binocular rivalry provides a clear and dramatic switch in awareness it suffers from the problem that the timing of these switches are unpredictable (though they follow a well-described probability distribution). An alternative paradigm, flash suppression (Wilke, Logothetis, \& Leopold, 2003; Wolfe, 1984) and its close relative, continuous flash suppression (Tsuchiya \& Koch, 2005), provide the ability to present a stimulus to one retina and render it entirely invisible by simultaneously presenting a different, salient stimulus to the other retina. In the original description of this paradigm (Wilke et al., 2003; Wolfe, 1984) the stimulus that will become invisible (the 'unaware' stimulus) is first presented for several seconds to one eye while a neutral background is presented to the other eye. This leads to adaptation of the neural representation of the target stimulus. The target stimulus is then rendered invisible by the sudden presentation of a high-intensity stimulus to the other eye. This stimulus normally consists of highcontrast colored squares ('Mondrians') or a field of moving dots. This paradigm yields a very reliable and complete disappearance of the target stimulus for several seconds until a rivalrous state resumes. This paradigm was developed further into 'continuous flash suppression' (Tsuchiya \& Koch, 2005). In this version a stream of continuously changing Mondrians is presented to one-eye. A low-intensity stimulus is then presented to the other eye for a brief period and remains entirely invisible. In this way a stream of stimuli can be presented to one eye without the observer ever being aware of them. This allows researchers to determine the fate of the neural representation of the unaware stimulus using electrophysiological or imaging techniques.

One factor that complicates the interpretation of binocular rivalry and flash suppression studies is the requirement for monocular presentation of the stimulus and the presence of the competing stimulus in the other eye. This problem is avoided in the motion-induced blindness (MIB) paradigm. In MIB studies large, binocularly presented, high-contrast shapes (bright yellow circles in the original description) 
spontaneously disappear from view when they are surrounded by a field of coherently moving dots (Bonneh, Cooperman, \& Sagi, 2001). The dots are also presented binocularly and never overlap with the circle and yet observers report the spontaneous disappearance of one, or more, of the circles, sometimes for seconds at a time. Several studies have used this paradigm to address the question of how the neural representations of the circles change when observers report their disappearance from awareness.

\subsubsection{Masking}

Another class of paradigms renders stimuli invisible by limiting the duration of processing of the stimulus or by exploiting the strong inhibitory connections that exist between the representations of nearby objects. Collectively known as masking paradigms, these approaches vary substantially in their implementation, i.e., whether the mask was presented before or after the stimulus (or both) and whether the mask spatially overlaps the stimulus, shares borders with it or simply surrounds it. These different forms of masking are likely to render the masked stimulus invisible through different mechanisms. For example backwards masking, in which a high-contrast pattern is presented shortly after the stimulus offset, has been suggested to work through preventing recurrent processing of the masked stimulus (Fahrenfort, Scholte, \& Lamme, 2007; Lamme, Zipser, \& Spekreijse, 2002). On the other hand, simultaneous contrast masking, in which a pattern is presented at the same time as the target stimulus but does not overlap spatially, likely works through suppressive connections between the mask and the unaware stimulus (Macknik, 2006). For foveal vision, contrast masking is highly similar to a phenomenon called visual crowding in which the conscious perception of an object is impaired by presenting it with accompanying flanker stimuli (Levi, 2008; Pelli \& Tillman, 2008; Whitney \& Levi, 2011). In peripheral vision however, the spatial characteristics of crowding and masking are rather different suggesting that crowding is in fact a distinct mechanism (Levi, 2008). Furthermore, while contrast masking appears to erase a stimulus from awareness by preventing feature detection, the presence of a crowded object can generally be detected. Its specific characteristics however are not recognized, presumably as a result of excessive feature integration between the target stimulus and the flankers (Pelli, Palomares, \& Majaj, 2004), a process that seems to occur after the flankers are consciously perceived (Wallis \& Bex, 2011).

Masking studies are particularly interesting for two reasons. Firstly many studies have varied the parameters of the mask to attempt to infer what aspect(s) of the neural representation of the masked stimulus is (are) necessary for awareness. Secondly masking studies have been used to study the neural networks that can be activated by a masked (and unconscious) stimulus. These latter studies have revealed that the masked stimulus can activate areas throughout the visual hierarchy up to and including motor output areas (Dehaene et al., 1998; Moutoussis \& Zeki, 2002) (albeit at lower intensities than an unmasked stimulus). The depth of processing of the masked stimulus can also be inferred by subsequent priming effects. For example a masked word can prime observers on a sentence completion task, meaning that some semantic processing 
of the mask has occurred, even though the observer remains completely unaware of the masked prime (Dehaene et al., 1998, 2001; Greenwald, Draine, \& Abrams, 1996; Marcel, 1983; Nakamura, Dehaene, Jobert, Le Bihan, \& Kouider, 2007).

\subsubsection{Attentional paradigms}

Patients with damage to the right parietal and temporoparietal cortex often suffer from hemispatial neglect. This condition is characterized by an inability to orient attention towards the contralateral (usually left) visual field. Patients with such a condition are often capable of perceiving stimuli presented to the neglected hemifield if they are presented in isolation, however in the presence of a competing stimulus in the normal hemifield patients often fail to report stimuli in the neglected field (Driver \& Mattingley, 1998; Rees et al., 2000; Rees, Wojciulik, et al., 2002). These findings were the original impetus behind arguments that attention is a necessary requirement for visual awareness. This argumentation was leant further support through the development of the change-blindness paradigm (Intraub, 1997; O'Regan, Rensink, \& Clark, 1999; Rensink, O’Regan, \& Clark, 1997; Simons \& Levin, 1997; Simons \& Rensink, 2005). In the original version of this paradigm observers view two sequentially presented visual scenes separated briefly by a blank screen. In one of the two scenes a change is introduced such as the removal of an object, or a change in color of a region of the scene. Surprisingly, observers fail to report the change, even after many repeated viewings of the scenes. The intervening blank period is critical, as it masks the onset transients, which would otherwise immediately reveal the location of the change. Initially this paradigm was argued to constitute evidence that we are only aware of stimuli that we attend to. When presented with the alternating visual scenes we randomly move our attention around the scene and we only become aware of the change if we happen to attend to the changing object. However an alternative interpretation would be that we are aware of much more of the content of the visual scene than just the region/object we are attending to (as would be our subjective experience), and that we are only able to encode attended items into working memory, allowing us to complete the task. Perhaps a more direct demonstration of the importance of attention for awareness comes from inattentional blindness paradigms (Mack, 2003; Mack \& Rock, 1998). Observers can miss a very salient item in a visual scene if their attention is directed to other features in the scene. This was dramatically demonstrated by the now famous 'Gorillas in our midst' paradigm in which observers often miss the presence of a man in a Gorilla suit that walks through two teams playing basketball if they are instructed to attend to the team playing in white (Simons \& Chabris, 1999). Such a paradigm gets to the heart of the role of attention in awareness. Are observers phenomenally aware of the Gorilla, but unable to report its presence? Or does diverting attention away from a stimulus render observers completely unaware of its presence?

The final paradigm that we will consider that manipulates awareness through manipulating attention is the attentional blink paradigm (Broadbent \& Broadbent, 1987; Raymond, Shapiro, \& Arnell, 1992). In a typical attentional blink task observers

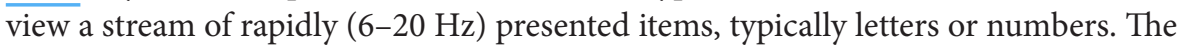


observer must perform two tasks, for example to press a button immediately as soon as the letter ' $\mathrm{X}$ ' appears, and to report whether a presented number was odd or even at the end of the trial. The critical manipulation is the time delay between the appearance of the ' $\mathrm{X}$ ' and the appearance of the number, the stimulus onset asynchrony (SOA). If the number is presented at a long SOA then observers have no trouble reporting the number. If however the number was presented at a short SOA then performance on the number task can fall to chance levels. Notably this does not occur if the number is presented immediately after the ' $\mathrm{X}$ ', a phenomenon known as 'lag 1 sparing' (Potter, Chun, Banks, \& Muckenhoupt, 1998). The attentional blink has mostly been used as a behavioral paradigm with the aim being to understand the mechanisms that lead to the reduced performance at short SOAs. A few studies have also used the attentional blink paradigm as a form of masking to render stimuli unaware and study the representation of the 'blinked' stimulus (Martens \& Wyble, 2010).

\subsubsection{Memory paradigms}

In experiments that require observers to report perceptual experience, the report is often given after the experience. This means that some form of memory about the preceding perceptual experience is involved in the probing process. This memory element can be investigated by extending the interval between the experience and the report and by adding additional stimuli or tasks in this interval period. Interestingly, the perceptual characteristics of visual short-term memory (Figure 3) may reveal important aspects of the mechanisms that give rise to conscious visual experiences (Crick \& Koch, 1990; Lamme, 2004). Distinct phases of visual short term memory can be distinguished. A brief ( $<0.5 \mathrm{~s})$ iconic memory contains a rich representation of recent visual experience (Sperling, 1960), while a much longer (seconds to minutes) visual working memory only maintains a very limited subset of about four representations (Luck \& Vogel, 1997). The transition from iconic to working memory depends on attention and subsequent visual stimulation. In the absence of subsequent stimulation, the rich information in iconic memory transfers to a fragile but rich form of longer lasting (seconds) memory from which attention can select a number of objects and features that far surpasses the classic capacity of working memory (Landman, Spekreijse, \& Lamme, 2003; Sligte, Scholte, \& Lamme, 2008; Sligte, Wokke, Tesselaar, Scholte, \& Lamme, 2010; Vandenbroucke, Sligte, \& Lamme, 2011). This fragile shortterm memory is however erased by new visual stimulation (Pinto, Sligte, Shapiro, \& Lamme, 2013; Sligte, Scholte, \& Lamme, 2009), leaving an observer with only the content of working memory from which to report. The attention-gated promotion of elements of a weak but rich visual representation to cognitive report systems strongly resembles the theoretical construct of the attention-gated transition from phenomenal to access consciousness making these memory paradigms an interesting approach to sample the mechanisms of visual consciousness (Crick \& Koch, 1990; Kouider, de Gardelle, Sackur, \& Dupoux, 2010; Lamme, 2010). 

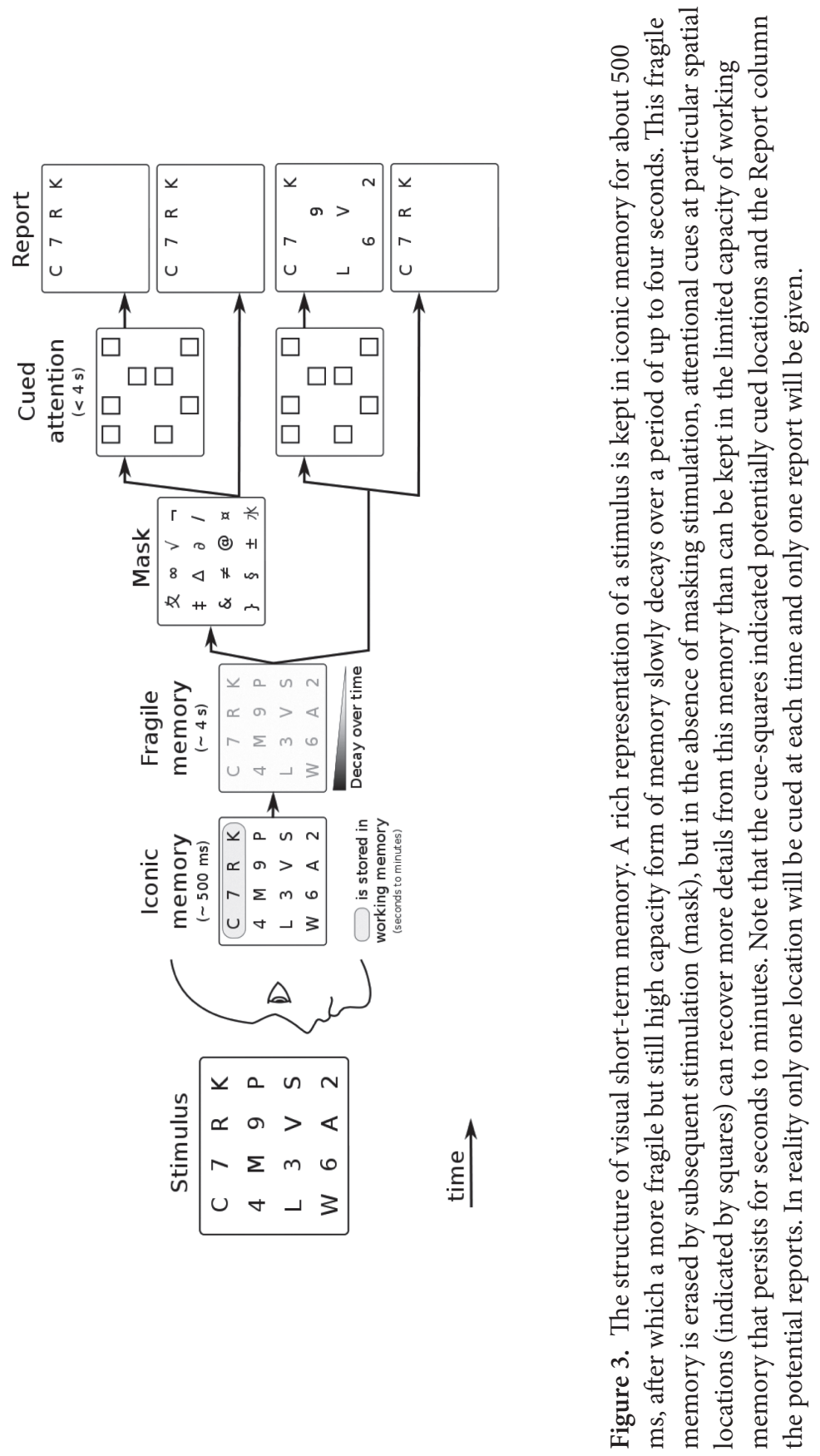


\subsubsection{Other paradigms}

Here we have focused on visual studies which have searched for the NCC by keeping the stimulation of the retina as similar as possible while varying the contents of the observers' visual consciousness (Kim \& Blake, 2005). There are many other approaches available to study consciousness for which we do not have space to cover in detail here but which we will briefly address. An alternative approach to studying awareness is to make changes to the global state of consciousness of the subject. An obvious example is anesthesia, which completely removes awareness of the environment. However as anesthesia globally alters brain activity it may be difficult to identify the critical neural processes that lead to awareness. Similar arguments can be made for studies that have examined the brain-activity patterns of subjects in a persistent vegetative state (PVS). Recent studies have demonstrated that some patients in PVS, though apparently completely unresponsive to external stimuli, are able to perform visual imagery experiments as assessed by the patterns of brain activity they produce in functional magnetic resonance imaging studies (Owen \& Coleman, 2008; Owen et al., 2006). These results have of course great consequences for the treatment of patients in PVS, but also for our understanding of awareness (see Charland-Verville, Vanhaudenhuyse, Laureys, \& Gosseries, this volume). These apparently unresponsive patients can follow complex instructions and change their brain activity in a manner similar to normal subjects. This result suggests a dissociation between the neural systems that enable motor-output and those that result in awareness of the environment. Similar dissociations also occur in rapid eye movement (REM) sleep states, where some form of visual awareness (which may be more akin to visual imagery) may be present yet motor output is inhibited (except eye movements) (Braun et al., 1998). Studying these states is of course extremely difficult because, even though significant progress is being made (Horikawa, Tamaki, Miyawaki, \& Kamitani, 2013), we still lack a method to reliably assess the contents of awareness in these cases. Similar problems exist for studies of visual imagery itself: the experimenter has little control over the exact contents of the participant's awareness making it difficult to assess the underlying neural mechanisms which lead to the formation of mental images. Nevertheless imaging studies have provided evidence that visual imagery reactivates similar areas of cortex that are activated by physical stimulation of the retina (Klein, Paradis, Poline, Kosslyn, \& Le Bihan, 2000; Knauff, Kassubek, Mulack, \& Greenlee, 2000; Kosslyn, Thompson, Kim, \& Alpert, 1995).

\section{Conclusion}

This overview of the most prominent current theories and methods in the scientific study of consciousness illustrates the large interest in the neurobiological basis of consciousness. Even though the presented theories differ on numerous aspects, there appears to be some consensus on the type of neural mechanisms that are important 
for the establishment of conscious experience. Notwithstanding the ongoing discussion as to when a neuronal representation of a visual scene transfers from being unconscious to conscious, the general scheme of broad and rich neuronal representations that require integration to become accessible for other cognitive systems is a recurrent feature of many theories. In addition to ongoing theoretical discussions, the field may benefit from continuing the recent neuroscientific approach to consciousness based on experimentation and data. By centering the scientific study of consciousness on its measurable underlying neural mechanisms we may be able to avoid misinterpretations of theoretical terminology and exploit the functional overlap between existing theories. Theoretical insights on the nature of consciousness will still be very useful in guiding the proposed experimental efforts, but constraining future (iterations of) theories with the growing body of data seems to be the most promising way to come to a true understanding of the neurobiology of consciousness (Lamme, 2006, 2010).

\section{References}

Arnold, D. H., Clifford, C. W., \& Wenderoth, P. (2001). Asynchronous processing in vision: Color leads motion. Current Biology, 11(8), 596-600. DOI: 10.1016/S0960-9822(01)00156-7

Azzopardi, P., \& Cowey, A. (1997). Is blindsight like normal, near-threshold vision? Proceedings of the National Academy of Sciences USA, 94(25), 14190-14194.

DOI: $10.1073 /$ pnas.94.25.14190

Baars, B. J. (1993). A cognitive theory of consciousness. New York, NY: Cambridge University Press.

Baars, B. J. (1997). In the theater of consciousness: The workspace of the mind. New York, NY: Oxford University Press. DOI: 10.1093/acprof:oso/9780195102659.001.1

Blake, R., \& Logothetis, N. K. (2002). Visual competition. Nature Reviews Neuroscience, 3(1), 13-21. DOI: $10.1038 / \mathrm{nrn} 701$

Block, N. (1995). On a confusion about a function of consciousness. Behavioral and Brain Sciences, 18(2), 227-287. DOI: 10.1017/S0140525X00038188

Block, N. (1996). How can we find the neural correlate of consciousness? Trends in Neurosciences, 19(11), 456-459. DOI: 10.1016/S0166-2236(96)20049-9

Block, N. (2005a). Two neural correlates of consciousness. Trends in Cognitive Sciences, 9(2), 46-52. DOI: 10.1016/j.tics.2004.12.006

Block, N. (2005b). The merely verbal problem of consciousness. Trends in Cognitive Sciences, 9(6), 270. DOI: 10.1016/j.tics.2005.04.009

Block, N. (2011). Perceptual consciousness overflows cognitive access. Trends in Cognitive Sciences, 15(12), 567-575. DOI: 10.1016/j.tics.2011.11.001

Bonneh, Y.S., Cooperman, A., \& Sagi, D. (2001). Motion-induced blindness in normal observers. Nature, 411(6839), 798-801. DOI: 10.1038/35081073

Born, R. T., \& Bradley, D. C. (2005). Structure and function of visual area MT. Annual Review of Neuroscience, 28, 157-189. DOI: 10.1146/annurev.neuro.26.041002.131052 
Bosman, C.A., Schoffelen, J.-M., Brunet, N., Oostenveld, R., Bastos, A. M., Womelsdorf, T., Rubehn, B., Stieglitz, T., De Weerd, P., \& Fries, P. (2012). Attentional stimulus selection through selective synchronization between monkey visual areas. Neuron, 75(5), 875-888. DOI: 10.1016/j.neuron.2012.06.037

Braun, A. R., Balkin, T. J., Wesensten, N. J., Gwadry, F., Carson, R.E., Varga, M., Baldwin, P., Belenky, G., \& Herscovitch, P. (1998). Dissociated pattern of activity in visual cortices and their projections during human rapid eye movement sleep. Science, 279(5347), 91-95. DOI: $10.1126 /$ science.279.5347.91

Broadbent, D.E., \& Broadbent, M.H. (1987). From detection to identification: Response to multiple targets in rapid serial visual presentation. Perception \& Psychophysics, 42(2), 105-113. DOI: $10.3758 / \mathrm{BF} 03210498$

Carrasco, M., Ling, S., \& Read, S. (2004). Attention alters appearance. Nature Neuroscience, 7(3), 308-313. DOI: $10.1038 / \mathrm{nn} 1194$

Chalmers, D. J. (1995). Facing up to the problem of consciousness. Journal of Consciousness Studies, 2(3), 200-219.

Chalmers, D. J. (1996). The conscious mind: In search of a fundamental theory. New York, NY: Oxford University Press.

Cohen, M. A., Cavanagh, P., Chun, M. M., \& Nakayama, K. (2012). The attentional requirements of consciousness. Trends in Cognitive Sciences, 16(8), 411-417. DOI: $10.1016 /$ j.tics.2012.06.013

Cohen, M. A., \& Dennett, D.C. (2011). Consciousness cannot be separated from function. Trends in Cognitive Sciences, 15(8), 358-364. DOI: 10.1016/j.tics.2011.06.008

Crick, F., \& Koch, C. (1990). Towards a neurobiological theory of consciousness. Seminars in the Neurosciences, 2, 263-275. DOI: 10.1016/B978-0-12-185254-2.50021-8

Crick, F., \& Koch, C. (1995). Are we aware of neural activity in primary visual cortex? Nature, 375(6527), 121-123. DOI: $10.1038 / 375121 \mathrm{a} 0$

Crick, F., \& Koch, C. (1998). Consciousness and neuroscience. Cerebral Cortex, 8(2), 97-107. DOI: $10.1093 /$ cercor/8.2.97

Crick, F., \& Koch, C. (2003). A framework for consciousness. Nature Neuroscience, 6(2), 119126. DOI: $10.1038 / \mathrm{nn} 0203-119$

Dehaene, S., Changeux, J.-P., Naccache, L., Sackur, J., \& Sergent, C. (2006). Conscious, preconscious, and subliminal processing: A testable taxonomy. Trends in Cognitive Sciences, 10(5), 204-211. DOI: 10.1016/j.tics.2006.03.007

Dehaene, S., Kerszberg, M., \& Changeux, J.-P. (1998). A neuronal model of a global workspace in effortful cognitive tasks. Proceedings of the National Academy of Sciences USA, 95(24), 14529-14534. DOI: 10.1073/pnas.95.24.14529

Dehaene, S., \& Naccache, L. (2001). Towards a cognitive neuroscience of consciousness: Basic evidence and a workspace framework. Cognition, 79(1-2), 1-37. DOI: 10.1016/S0010-0277(00)00123-2

Dehaene, S., Naccache, L., Cohen, L., Bihan, D. L., Mangin, J. F., Poline, J. B., \& Rivière, D. (2001). Cerebral mechanisms of word masking and unconscious repetition priming. Nature $\mathrm{Neu}$ roscience, 4(7), 752-758. DOI: 10.1038/89551

Dehaene, S., Naccache, L., Le Clec'H, G., Koechlin, E., Mueller, M., Dehaene-Lambertz, G., van de Moortele, P.F., \& Le Bihan, D. (1998). Imaging unconscious semantic priming. Nature, 395(6702), 597-600. DOI: 10.1038/26967

Driver, J., \& Mattingley, J. B. (1998). Parietal neglect and visual awareness. Nature Neuroscience, 1(1), 17-22. DOI: $10.1038 / 217$

(C) 2015. John Benjamins Publishing Company

All rights reserved 
Edelman, G. M. (1987). Neural darwinism: The theory of neuronal group selection. New York, NY: Basic Books.

Edelman, G. M. (1993). Neural darwinism: Selection and reentrant signaling in higher brain function. Neuron, 10(2), 115-125. DOI: 10.1016/0896-6273(93)90304-A

Edelman, G. M., Gally, J. A., \& Baars, B. J. (2011). Biology of consciousness. Frontiers in Psychology, 2, 4. DOI: 10.3389/fpsyg.2011.00004

Edelman, G. M., \& Mountcastle, V.B. (1978). The mindful brain: Cortical organization and the group-selective theory of higher brain function. Cambridge, MA: MIT Press.

Edelman, G. M., \& Tononi, G. (2000). A universe of consciousness: How matter becomes imagination. New York, NY: Basic Books.

Fahrenfort, J. J., \& Lamme, V.A.F. (2012). A true science of consciousness explains phenomenology: Comment on Cohen and Dennett. Trends in Cognitive Sciences, 16(3), 138-139. DOI: 10.1016/j.tics.2012.01.004

Fahrenfort, J. J., Scholte, H.S., \& Lamme, V. A. F. (2007). Masking disrupts reentrant processing in human visual cortex. Journal of Cognitive Neuroscience, 19(9), 1488-1497.

DOI: 10.1162/jocn.2007.19.9.1488

Felleman, D., \& Van Essen, D.C. (1991). Distributed hierarchical processing in the primate cerebral cortex. Cerebral Cortex, 1(1), 1-47. DOI: 10.1093/cercor/1.1.1

Ferrera, V.P., Rudolph, K. K., \& Maunsell, J.H. (1994). Responses of neurons in the parietal and temporal visual pathways during a motion task. Journal of Neuroscience, 14(10), 6171-6186.

Gaillard, R., Dehaene, S., Adam, C., Clémenceau, S., Hasboun, D., Baulac, M., Cohen, L., \& Nacchache, L. (2009). Converging intracranial markers of conscious access. PLoS Biology, 7(3), e61. DOI: 10.1371/journal.pbio.1000061

Galvin, S. J., Podd, J. V, Drga, V., \& Whitmore, J. (2003). Type 2 tasks in the theory of signal detectability: Discrimination between correct and incorrect decisions. Psychonomic Bulletin \& Review, 10(4), 843-876. DOI: 10.3758/BF03196546

Goodale, M.A. (2004). An evolving view of duplex vision: Separate but interacting cortical pathways for perception and action. Current Opinion in Neurobiology, 14(2), 203-211. DOI: 10.1016/j.conb.2004.03.002

Goodale, M. A., \& Milner, A. (1992). Separate visual pathways for perception and action. Trends in Neurosciences, 15(1), 20-25. DOI: 10.1016/0166-2236(92)90344-8

Goodale, M. A., Milner, A. D., Jakobson, L. S., \& Carey, D. P. (1991). A neurological dissociation between perceiving objects and grasping them. Nature, 349(6305), 154-156.

DOI: $10.1038 / 349154 \mathrm{a} 0$

Greenwald, A. G., Draine, S. C., \& Abrams, R. L. (1996). Three cognitive markers of unconscious semantic activation. Science, 273(5282), 1699-1702. DOI: 10.1126/science.273.5282.1699

Grill-Spector, K., \& Malach, R. (2004). The human visual cortex. Annual Review of Neuroscience, 27, 649-677. DOI: 10.1146/annurev.neuro.27.070203.144220

Hameroff, S. (2006a). The entwined mysteries of anesthesia and consciousness: Is there a common underlying mechanism? Anesthesiology, 105(2), 400-412.

DOI: 10.1097/00000542-200608000-00024

Hameroff, S. (2006b). Consciousness, neurobiology and quantum mechanics: The case for a connection. In J. A. Tuszynski (Ed.), The emerging physics of consciousness (pp. 193-253). Berlin: Springer-Verlag. DOI: 10.1007/3-540-36723-3_6

Hopfinger, J.B., Buonocore, M.H., \& Mangun, G. R. (2000). The neural mechanisms of topdown attentional control. Nature Neuroscience, 3(3), 284-291. DOI: 10.1038/72999

(C) 2015. John Benjamins Publishing Company

All rights reserved 
Horikawa, T., Tamaki, M., Miyawaki, Y., \& Kamitani, Y. (2013). Neural decoding of visual imagery during sleep. Science, 340(6132), 639-642. DOI: 10.1126/science.1234330

Intraub, H. (1997). The representation of visual scenes. Trends in Cognitive Sciences, 1(6), 217 222. DOI: $10.1016 /$ S1364-6613(97)01067-X

Jackendoff, R. (1996). How language helps us think. Pragmatics \& Cognition, 4(1), 1-34. DOI: $10.1075 / \mathrm{pc} .4 .1 .03 \mathrm{jac}$

James, T. W., Culham, J., Humphrey, G.K., Milner, A. D., \& Goodale, M.A. (2003). Ventral occipital lesions impair object recognition but not object-directed grasping: An fMRI study. Brain, 126(11), 2463-2475. DOI: 10.1093/brain/awg248

Kim, C., \& Blake, R. (2005). Psychophysical magic: Rendering the visible "invisible". Trends in Cognitive Sciences, 9(8), 381-388. DOI: 10.1016/j.tics.2005.06.012

Klein, I., Paradis, A.-L., Poline, J. B., Kosslyn, S. M., \& Le Bihan, D. (2000). Transient activity in the human calcarine cortex during visual-mental imagery: An event-related fMRI study. Journal of Cognitive Neuroscience, 12(Suppl. 2), 15-23. DOI: 10.1162/089892900564037

Knauff, M., Kassubek, J., Mulack, T., \& Greenlee, M. W. (2000). Cortical activation evoked by visual mental imagery as measured by fMRI. Neuroreport, 11(18), 3957-3962.

DOI: $10.1097 / 00001756-200012180-00011$

Ko, Y., \& Lau, H. (2012). A detection theoretic explanation of blindsight suggests a link between conscious perception and metacognition. Philosophical Transactions of the Royal Society of London B: Biological Sciences, 367(1594), 1401-1411. DOI: 10.1098/rstb.2011.0380

Koch, C. (2004). The quest for consciousness: A neurobiological approach. Englewood, CO: Roberts \& Company.

Koch, C., \& Hepp, K. (2006). Quantum mechanics in the brain. Nature, 440(7084), 611. DOI: $10.1038 / 440611 \mathrm{a}$

Koch, C., \& Tsuchiya, N. (2007). Attention and consciousness: Two distinct brain processes. Trends in Cognitive Sciences, 11(1), 16-22. DOI: 10.1016/j.tics.2006.10.012

Koch, C., \& Tsuchiya, N. (2012). Attention and consciousness: Related yet different. Trends in Cognitive Sciences, 16(2), 103-104. DOI: 10.1016/j.tics.2011.11.012

Kolb, F. C., \& Braun, J. (1995). Blindsight in normal observers. Nature, 377(6547), 336-338. DOI: $10.1038 / 377336 \mathrm{a} 0$

Kornbrot, D.E. (2006). Signal detection theory, the approach of choice: Model-based and distribution-free measures and evaluation. Perception \& Psychophysics, 68(3), 393-414. DOI: $10.3758 / \mathrm{BF} 03193685$

Kosslyn, S. M., Thompson, W.L., Kim, I. J., \& Alpert, N. M. (1995). Topographical representations of mental images in primary visual cortex. Nature, 378(6556), 496-498. DOI: $10.1038 / 378496 \mathrm{a} 0$

Kouider, S., De Gardelle, V., Sackur, J., \& Dupoux, E. (2010). How rich is consciousness? The partial awareness hypothesis. Trends in Cognitive Sciences, 14(7), 301-307. DOI: 10.1016/j.tics.2010.04.006

Kouider, S., \& Dehaene, S. (2007). Levels of processing during non-conscious perception: A critical review of visual masking. Philosophical Transactions of the Royal Society of London B: Biological Sciences, 362(1481), 857-875. DOI: 10.1098/rstb.2007.2093

Kunimoto, C., Miller, J., \& Pashler, H. (2001). Confidence and accuracy of near-threshold discrimination responses. Consciousness and Cognition, 10(3), 294-340. DOI: $10.1006 /$ ccog.2000.0494

Lamme, V.A.F. (1995). The neurophysiology of figure-ground segregation in primary visual cortex. Journal of Neuroscience, 15(2), 1605-1615. 
Lamme, V.A. F. (2003). Why visual attention and awareness are different. Trends in Cognitive Sciences, 7(1), 12-18. DOI: 10.1016/S1364-6613(02)00013-X

Lamme, V.A. F. (2004). Separate neural definitions of visual consciousness and visual attention: A case for phenomenal awareness. Neural Networks, 17(5-6), 861-872.

DOI: 10.1016/j.neunet. 2004.02.005

Lamme, V.A.F. (2006). Towards a true neural stance on consciousness. Trends in Cognitive Sciences, 10(11), 494-501. DOI: 10.1016/j.tics.2006.09.001

Lamme, V.A.F. (2010). How neuroscience will change our view on consciousness. Cognitive Neuroscience, 1(3), 204-220. DOI: 10.1080/17588921003731586

Lamme, V.A. F., \& Roelfsema, P. R. (2000). The distinct modes of vision offered by feedforward and recurrent processing. Trends in Neurosciences, 23(11), 571-579. DOI: 10.1016/S0166-2236(00)01657-X

Lamme, V. A. F., Supèr, H., \& Spekreijse, H. (1998). Feedforward, horizontal, and feedback processing in the visual cortex. Current Opinion in Neurobiology, 8(4), 529-535. DOI: $10.1016 /$ S0959-4388(98)80042-1

Lamme, V.A. F., Zipser, K., \& Spekreijse, H. (2002). Masking interrupts figure-ground signals in V1. Journal of Cognitive Neuroscience, 14(7), 1044-1053.

DOI: $10.1162 / 089892902320474490$

Landman, R., Spekreijse, H., \& Lamme, V.A.F. (2003). Large capacity storage of integrated objects before change blindness. Vision Research, 43(2), 149-164.

DOI: $10.1016 /$ S0042-6989(02)00402-9

Levi, D. M. (2008). Crowding - An essential bottleneck for object recognition: A mini-review. Vision Research, 48(5), 635-654. DOI: 10.1016/j.visres.2007.12.009

Levine, J. (1983). Materialism and qualia: The explanatory gap. Pacific Philosophical Quarterly, 64(4), 354-361.

Luck, S. J., \& Vogel, E. K. (1997). The capacity of visual working memory for features and conjunctions. Nature, 390(6657), 279-281. DOI: 10.1038/36846

Mack, A. (2003). Inattentional blindness: Looking without seeing. Current Directions in Psychological Science, 12(5), 180-184. DOI: 10.1111/1467-8721.01256

Mack, A., \& Rock, I. (1998). Inattentional blindness. Cambridge, MA: MIT Press.

Macknik, S. L. (2006). Visual masking approaches to visual awareness. Progress in Brain Research, 155, 177-215. DOI: 10.1016/S0079-6123(06)55011-3

Macmillan, N. A., \& Creelman, D. C. (1991). Detection theory: A user's guide. Cambridge, MA: Cambridge University Press.

Maniscalco, B., \& Lau, H. (2012). A signal detection theoretic approach for estimating metacognitive sensitivity from confidence ratings. Consciousness and Cognition, 21(1), 422-430. DOI: 10.1016/j.concog.2011.09.021

Marcel, A.J. (1983). Conscious and unconscious perception: Experiments on visual masking and word recognition. Cognitive Psychology, 15(2), 197-237.

DOI: 10.1016/0010-0285(83)90009-9

Marchetti, G. (2012). Against the view that consciousness and attention are fully dissociable. Frontiers in Psychology, 3, 36. DOI: 10.3389/fpsyg.2012.00036

Martens, S., \& Wyble, B. (2010). The attentional blink: Past, present, and future of a blind spot in perceptual awareness. Neuroscience and Biobehavioral Reviews, 34(6), 947-957.

DOI: $10.1016 /$ j.neubiorev.2009.12.005

Maunsell, J.H. R., \& Newsome, W.T. (1987). Visual processing in monkey extrastriate cortex. Annual Review of Neuroscience, 10, 363-401. DOI: 10.1146/annurev.ne.10.030187.002051

(C) 2015. John Benjamins Publishing Company

All rights reserved 
McFadden, J. (2002a). Synchronous firing and its influence on the brains electromagnetic field. Journal of Consciousness Studies, 9(4), 23-50.

McFadden, J. (2002b). The conscious electromagnetic information (Cemi) field theory: The hard problem made easy? Journal of Consciousness Studies, 9(8), 45-60.

Merigan, W.H., \& Maunsell, J.H. (1993). How parallel are the primate visual pathways? Annual Review of Neuroscience, 16, 369-402. DOI: 10.1146/annurev.ne.16.030193.002101

Merikle, P., Smilek, D., \& Eastwood, J. (2001). Perception without awareness: Perspectives from cognitive psychology. Cognition, 79(1-2), 115-134. DOI: 10.1016/S0010-0277(00)00126-8

Mesulam, M. M. (1998). From sensation to cognition. Brain, 121(6), 1013-1052. DOI: $10.1093 /$ brain/121.6.1013

Miller, S.M. (2001). Binocular rivalry and the cerebral hemispheres: With a note on the correlates and constitution of visual consciousness. Brain and Mind, 2(1), 119-149. DOI: 10.1023/A:1017981619014

Miller, S. M. (2007). On the correlation/constitution distinction problem (and other hard problems) in the scientific study of consciousness. Acta Neuropsychiatrica, 19(3), 159-176. DOI: $10.1111 / \mathrm{j} .1601-5215.2007 .00207 . \mathrm{x}$

Miller, S. M. (Ed.). (2013). The constitution of visual consciousness: Lessons from binocular rivalry. Advances in Consciousness Research (Vol. 90). Amsterdam, The Netherlands: John Benjamins Publishing Company. DOI: 10.1075/aicr.90

Milner, A., \& Goodale, M. A. (1993). Separate visual pathways for perception and action. Progress in Brain Research, 95, 317-337. DOI: 10.1016/S0079-6123(08)60379-9

Mishkin, M., \& Ungerleider, L. G. (1982). Contribution of striate inputs to the visuospatial functions of parieto-preoccipital cortex in monkeys. Behavioural Brain Research, 6(1), 57-77. DOI: 10.1016/0166-4328(82)90081-X

Moutoussis, K., \& Zeki, S. (1997a). A direct demonstration of perceptual asynchrony in vision. Proceedings of the Royal Society of London B: Biological Sciences, 264(1380), 393-399. DOI: $10.1098 / \mathrm{rspb} .1997 .0056$

Moutoussis, K., \& Zeki, S. (1997b). Functional segregation and temporal hierarchy of the visual perceptive systems. Proceedings of the Royal Society of London B: Biological Sciences, 264(1387), 1407-1414. DOI: 10.1098/rspb.1997.0196

Moutoussis, K., \& Zeki, S. (2002). The relationship between cortical activation and perception investigated with invisible stimuli. Proceedings of the National Academy of Sciences USA, 99(14), 9527-9532. DOI: 10.1073/pnas.142305699

Nagel, T. (1974). What is it like to be a bat? Philosophical Review, 83(4), 435-450. DOI: $10.2307 / 2183914$

Nakamura, K., Dehaene, S., Jobert, A., Le Bihan, D., \& Kouider, S. (2007). Task-specific change of unconscious neural priming in the cerebral language network. Proceedings of the National Academy of Sciences USA, 104(49), 19643-19648. DOI: 10.1073/pnas.0704487104

Oizumi, M., Albantakis, L., \& Tononi, G. (2014). From the phenomenology to the mechanisms of consciousness: Integrated Information Theory 3.0. PLoS Computational Biology, 10(5), e1003588. DOI: 10.1371/journal.pcbi.1003588

O’Regan, J.K., Rensink, R. A., \& Clark, J. J. (1999). Change-blindness as a result of "mudsplashes". Nature, 398(6722), 34. DOI: 10.1038/17953

Owen, A.M., \& Coleman, M.R. (2008). Detecting awareness in the vegetative state. Annals of the New York Academy of Sciences, 1129, 130-138. DOI: 10.1196/annals.1417.018 
Owen, A. M., Coleman, M. R., Boly, M., Davis, M.H., Laureys, S., \& Pickard, J.D. (2006). Detecting awareness in the vegetative state. Science, 313(5792), 1402.

DOI: $10.1126 /$ science.1130197

Pelli, D. G., Palomares, M., \& Majaj, N. J. (2004). Crowding is unlike ordinary masking: Distinguishing feature integration from detection. Journal of Vision, 4(12), 12. DOI: $10.1167 / 4.12 .12$

Pelli, D. G., \& Tillman, K. A. (2008). The uncrowded window of object recognition. Nature Neuroscience, 11(10), 1129-1135. DOI: 10.1038/nn.2187

Persaud, N., McLeod, P., \& Cowey, A. (2007). Post-decision wagering objectively measures awareness. Nature Neuroscience, 10(2), 257-261. DOI: 10.1038/nn1840

Pinto, Y., Sligte, I. G., Shapiro, K. L., \& Lamme, V. A. F. (2013). Fragile visual short-term memory is an object-based and location-specific store. Psychonomic Bulletin \& Review, 20(4), 732-739. DOI: 10.3758/s13423-013-0393-4

Pöppel, E., Brinkmann, R., Von Cramon, D., \& Singer, W. (1978). Association and dissociation of visual functions in a case of bilateral occipital lobe infarction. Archiv für Psychiatrie und Nervenkrankheiten, 225(1), 1-21. DOI: 10.1007/BF00367348

Pöppel, E., Held, R., \& Frost, D. (1973). Residual visual function after brain wounds involving the central visual pathways in man. Nature, 243(5405), 295-296. DOI: 10.1038/243295a0

Posner, M. I. (1994). Attention: The mechanisms of consciousness. Proceedings of the National Academy of Sciences USA, 91(16), 7398-7403. DOI: 10.1073/pnas.91.16.7398

Posner, M. I. (2012). Attentional networks and consciousness. Frontiers in Psychology, 3, 64. DOI: $10.3389 /$ fpsyg.2012.00064

Potter, M. C., Chun, M. M., Banks, B. S., \& Muckenhoupt, M. (1998). Two attentional deficits in serial target search: The visual attentional blink and an amodal task-switch deficit. Journal of Experimental Psychology: Learning, Memory, and Cognition, 24(4), 979-992. DOI: 10.1037/0278-7393.24.4.979

Price, N. S. C. (2013). Overview of visual system structure and function. In S. M. Miller (Ed.), The constitution of visual consciousness: Lessons from binocular rivalry (pp. 37-76). Advances in Consciousness Research (Vol. 90). Amsterdam, The Netherlands: John Benjamins Publishing Company. DOI: 10.1075/aicr.90.03pri

Raffone, A., \& Pantani, M. (2010). A global workspace model for phenomenal and access consciousness. Consciousness and Cognition, 19(2), 580-596.

DOI: 10.1016/j.concog.2010.03.013

Raymond, J.E., Shapiro, K. L., \& Arnell, K. M. (1992). Temporary suppression of visual processing in an RSVP task: An attentional blink? Journal of Experimental Psychology: Human Perception and Performance, 18(3), 849-860. DOI: 10.1037/0096-1523.18.3.849

Rees, G., Kreiman, G., \& Koch, C. (2002). Neural correlates of consciousness in humans. Nature Reviews Neuroscience, 3(4), 261-270. DOI: 10.1038/nrn783

Rees, G., Wojciulik, E., Clarke, K., Husain, M., Frith, C., \& Driver, J. (2000). Unconscious activation of visual cortex in the damaged right hemisphere of a parietal patient with extinction. Brain, 123(8), 1624-1633. DOI: 10.1093/brain/123.8.1624

Rees, G., Wojciulik, E., Clarke, K., Husain, M., Frith, C., \& Driver, J. (2002). Neural correlates of conscious and unconscious vision in parietal extinction. Neurocase, 8(5), 387-393. DOI: $10.1093 /$ neucas/8.5.387

Rensink, R. A., O’Regan, J.K., \& Clark, J. J. (1997). To see or not to see: The need for attention to perceive changes in scenes. Psychological Science, 8(5), 368-373.

DOI: $10.1111 /$ j.1467-9280.1997.tb00427.x

(C) 2015. John Benjamins Publishing Company

All rights reserved 
Revonsuo, A. (2001). Can functional brain imaging discover consciousness in the brain? Journal of Consciousness Studies, 8(3), 3-23.

Roe, A. W., Chelazzi, L., Connor, C. E., Conway, B. R., Fujita, I., Gallant, J. L., Lu, H., \& Vanduffel, W. (2012). Toward a unified theory of visual area V4. Neuron, 74(1), 12-29. DOI: 10.1016/j.neuron.2012.03.011

Roelfsema, P. R. (2006). Cortical algorithms for perceptual grouping. Annual Review of Neuroscience, 29, 203-227. DOI: 10.1146/annurev.neuro.29.051605.112939

Seidemann, E., Poirson, A. B., Wandell, B. A., \& Newsome, W.T. (1999). Color signals in area MT of the macaque monkey. Neuron, 24(4), 911-917. DOI: 10.1016/S0896-6273(00)81038-7

Seth, A. K. (2009). Explanatory correlates of consciousness: Theoretical and computational challenges. Cognitive Computation, 1(1), 50-63. DOI: 10.1007/s12559-009-9007-x

Seth, A. K. (2010). The grand challenge of consciousness. Frontiers in Psychology, 1, 5. DOI: $10.3389 /$ fpsyg.2010.00005

Simons, D. J., \& Chabris, C. F. (1999). Gorillas in our midst: Sustained inattentional blindness for dynamic events. Perception, 28(9), 1059-1074. DOI: 10.1068/p2952

Simons, D. J., \& Levin, D. T. (1997). Change blindness. Trends in Cognitive Sciences, 1(7), 261267. DOI: $10.1016 /$ S1364-6613(97)01080-2

Simons, D. J., \& Rensink, R.A. (2005). Change blindness: Past, present, and future. Trends in Cognitive Sciences, 9(1), 16-20. DOI: 10.1016/j.tics.2004.11.006

Sligte, I. G., Scholte, H. S., \& Lamme, V. A. F. (2008). Are there multiple visual short-term memory stores? PLoS ONE, 3(2), e1699. DOI: 10.1371/journal.pone.0001699

Sligte, I. G., Scholte, H. S., \& Lamme, V.A.F. (2009). V4 activity predicts the strength of visual short-term memory representations. Journal of Neuroscience, 29(23), 7432-7438. DOI: 10.1523/JNEUROSCI.0784-09.2009

Sligte, I. G., Wokke, M.E., Tesselaar, J. P., Scholte, H. S., \& Lamme, V. A. F. (2010). Magnetic stimulation of the dorsolateral prefrontal cortex dissociates fragile visual short-term memory from visual working memory. Neuropsychologia, 49(6), 1578-1588.

DOI: 10.1016/j.neuropsychologia.2010.12.010

Sperling, G. (1960). Negative afterimage without prior positive image. Science, 131(3413), 16131614. DOI: $10.1126 /$ science.131.3413.1613

Stoerig, P., \& Cowey, A. (2007). Blindsight. Current Biology, 17(19), R822-R824.

DOI: $10.1016 /$ j.cub.2007.07.016

Tononi, G. (2004). An information integration theory of consciousness. BMC Neuroscience, 5, 42. DOI: $10.1186 / 1471-2202-5-42$

Tononi, G. (2008). Consciousness as integrated information: A provisional manifesto. Biological Bulletin, 215(3), 216-242. DOI: 10.2307/25470707

Tononi, G., \& Edelman, G. M. (1998). Consciousness and complexity. Science, 282(5395), 18461851. DOI: $10.1126 /$ science.282.5395.1846

Tononi, G., \& Koch, C. (2008). The neural correlates of consciousness: An update. Annals of the New York Academy of Sciences, 1124, 239-261. DOI: 10.1196/annals.1440.004

Tononi, G., \& Sporns, O. (2003). Measuring information integration. BMC Neuroscience, 4, 31. DOI: $10.1186 / 1471-2202-4-31$

Tovee, M. J. (1994). How fast is the speed of thought? Current Biology, 4(12), 1125-1127. DOI: $10.1016 / \mathrm{S} 0960-9822(00) 00253-0$

Treue, S. (2003). Visual attention: The where, what, how and why of saliency. Current Opinion in Neurobiology, 13(4), 428-432. DOI: 10.1016/S0959-4388(03)00105-3

(C) 2015. John Benjamins Publishing Company

All rights reserved 
Tsuchiya, N., \& Koch, C. (2005). Continuous flash suppression reduces negative afterimages. Nature Neuroscience, 8(8), 1096-1101. DOI: 10.1038/nn1500

van Boxtel, J. J.A., Tsuchiya, N., \& Koch, C. (2010a). Consciousness and attention: On sufficiency and necessity. Frontiers in Psychology, 1, 217. DOI: 10.3389/fpsyg.2010.00217

van Boxtel, J.J.A., Tsuchiya, N., \& Koch, C. (2010b). Opposing effects of attention and consciousness on afterimages. Proceedings of the National Academy of Sciences USA, 107(19), 8883-8888. DOI: 10.1073/pnas.0913292107

Vandenbroucke, A. R., Sligte, I. G., \& Lamme, V. A. F. (2011). Manipulations of attention dissociate fragile visual short-term memory from visual working memory. Neuropsychologia, 49(6), 1559-1568. DOI: 10.1016/j.neuropsychologia.2010.12.044

Wallis, T.S. A., \& Bex, P. J. (2011). Visual crowding is correlated with awareness. Current Biology, 21(3), 254-258. DOI: 10.1016/j.cub.2011.01.011

Watanabe, M., Cheng, K., Murayama, Y., Ueno, K., Asamizuya, T., Tanaka, K., \& Logothetis, N. (2011). Attention but not awareness modulates the BOLD signal in the human V1 during binocular suppression. Science, 334(6057), 829-831. DOI: 10.1126/science.1203161

Weiskrantz, L., Barbur, J. L., \& Sahraie, A. (1995). Parameters affecting conscious versus unconscious visual discrimination with damage to the visual cortex (V1). Proceedings of the National Academy of Sciences USA, 92(13), 6122-6126. DOI: 10.1073/pnas.92.13.6122

Weiskrantz, L., Warrington, E. K., Sanders, M.D., \& Marshall, J. (1974). Visual capacity in the hemianopic field following a restricted occipital ablation. Brain, 97(4), 709-728. DOI: $10.1093 /$ brain/97.1.709

Whitney, D., \& Levi, D. M. (2011). Visual crowding: A fundamental limit on conscious perception and object recognition. Trends in Cognitive Sciences, 15(4), 160-168. DOI: 10.1016/j.tics.2011.02.005

Wilke, M., Logothetis, N. K., \& Leopold, D. A. (2003). Generalized flash suppression of salient visual targets. Neuron, 39(6), 1043-1052. DOI: 10.1016/j.neuron.2003.08.003

Wolfe, J.M. (1984). Reversing ocular dominance and suppression in a single flash. Vision Research, 24(5), 471-478. DOI: 10.1016/0042-6989(84)90044-0

Zeki, S. (2001). Localization and globalization in conscious vision. Annual Review of Neuroscience, 24, 57-86. DOI: 10.1146/annurev.neuro.24.1.57

Zeki, S. (2008). The disunity of consciousness. Progress in Brain Research, 168, 11-18. DOI: 10.1016/S0079-6123(07)68002-9

Zeki, S., \& Bartels, A. (1998a). The autonomy of the visual systems and the modularity of conscious vision. Philosophical Transactions of the Royal Society of London B: Biological Sciences, 353(1377), 1911-1914. DOI: 10.1098/rstb.1998.0343

Zeki, S., \& Bartels, A. (1998b). The asynchrony of consciousness. Proceedings of the Royal Society of London B: Biological Sciences, 265(1405), 1583-1585. DOI: 10.1098/rspb.1998.0475

Zeki, S., \& ffytche, D.H. (1998). The Riddoch syndrome: Insights into the neurobiology of conscious vision. Brain, 121(1), 25-45. DOI: 10.1093/brain/121.1.25

Zipser, K., Lamme, V.A. F., \& Schiller, P.H. (1996). Contextual modulation in primary visual cortex. Journal of Neuroscience, 16(22), 7376-7389. 